\title{
Moving in a Moving World: A Review on Vestibular Motion Sickness
}

\author{
Giovanni Bertolini* and Dominik Straumann \\ Department of Neurology, University Hospital Zurich, Zurich, Switzerland
}

Motion sickness is a common disturbance occurring in healthy people as a physiological response to exposure to motion stimuli that are unexpected on the basis of previous experience. The motion can be either real, and therefore perceived by the vestibular system, or illusory, as in the case of visual illusion. A multitude of studies has been performed in the last decades, substantiating different nauseogenic stimuli, studying their specific characteristics, proposing unifying theories, and testing possible countermeasures. Several reviews focused on one of these aspects; however, the link between specific nauseogenic stimuli and the unifying theories and models is often not clearly detailed. Readers unfamiliar with the topic, but studying a condition that may involve motion sickness, can therefore have difficulties to understand why a specific stimulus will induce motion sickness. So far, this general audience struggles to take advantage of the solid basis provided by existing theories and models. This review focuses on

OPEN ACCESS

Edited by: Sergio Carmona Instituto de Neurociencias de Buenos Aires, Argentina

Reviewed by: Bernard Cohen, Mount Sinai School of Medicine, USA Sergei B. Yakushin, Mount Sinai School of Medicine, USA

*Correspondence:

Giovanni Bertolin giovanni.bertolini@usz.ch

Specialty section: This article was submitted to Neuro-otology, a section of the journal

Frontiers in Neurology

Received: 05 November 2015 Accepted: 01 February 2016 Published: 15 February 2016

Citation:

Bertolini G and Straumann D (2016) Moving in a Moving World: A Review on Vestibular Motion Sickness.

Front. Neurol. 7:14. doi: 10.3389/fneur.2016.00014 vestibular-only motion sickness, listing the relevant motion stimuli, clarifying the sensory signals involved, and framing them in the context of the current theories.

Keywords: motion sickness, vestibular, sensory conflict, cross-coupling stimulus, linear oscillations

\section{INTRODUCTION}

Motion sickness $(1,2)$ is a syndrome elicited in healthy subjects by ongoing passive self-motion that contains certain dynamic and kinematic properties (see Properties of Nauseogenic Stimuli). Illusions of such passive self-motion, as induced by moving visual surrounds, may also produce this condition (2). The first considerations on motion sickness known to us date back more than 2000 years, when the Greek physician Hippocrates observed that "sailing on the seas proves that motion disorders the body." In those ancient times, boats provided one of the only few forms of passive motion to which individuals were exposed. Because passive motion (car, bus, train, and plane) and illusion of passive motion (video games on large screens, 3D movies, and virtual reality) are now abundant in modern life, motion sickness has become a frequent problem (3-8). As the time spent on transport systems occupies a considerable part of daily life, travelers normally perform a variety of activities while being transported, leading to various active head movements during passive motion. However, motion sickness can be provoked or aggravated by active head movements in the presence of passive motion (8-12), considerably hindering the quality of travel.

Depending on its severity, the syndrome of motion sickness consists of various combinations of the following signs and symptoms: drowsiness, dizziness, discomfort, restiveness, repetitive yawning, stomach awareness, nausea, pallor, sweating, headache, malaise, bradycardia, arterial hypotension vomiting, and apathy $(2,12,13)$. Susceptibility to motion sickness varies considerably among subjects (14-19), whereby genetic factors and age play an important role (20,21). Notably, there is a strong association between the susceptibility to motion sickness and migraine (22-29). 


\section{PROPERTIES OF NAUSEOGENIC STIMULI}

Sine qua non for developing motion sickness is exposure to a real or illusory motion stimulus (2). Subject without labyrinthine vestibular function do not become motion sick (30-32); thus, the vestibular system appears to always take part in a nauseogenic stimulus. One may argue, however, that motion sickness can also be caused by stimuli that do not activate the labyrinth such as visual illusion of motion $(8,33-35)$. To understand the link between these two apparently distinct provocative stimuli, it is important to consider that the vestibular system is constantly involved in the perception of self-motion (36), as the brain continuously takes the vestibular input into account. Overall, it is possible to assert that motion sickness is occurring whenever the subjects are exposed to stimuli causing conflicts between motionsensitive input signals $(2,13)$.

The motion-sensitive inputs to our nervous system originate from different sensory systems (mainly vestibular, but also visual and somatosensory). Each system has its sensory-specific sensitivity, optimized to detect different aspects of the motion stimuli $(36,37)$. Vision, for example, cannot distinguish the effect of self-motion from the actual motion in the observed scene (e.g., feelings of illusory movement when looking at a moving train from a window seat) and becomes less reliable as the light dims. Within the vestibular organs, the semicircular canals, working as gyroscopes, inform us when our own angular velocity changes, but they are unable to report constant-velocity rotation; the otolith organs, in turn, measure the direction of accelerations, but, as any accelerometer, they cannot distinguish between gravity and inertial forces (38). In the brainstem and cerebellum, all sensory signals are merged, weighing them according to their reliability in a process optimized to obtain the best estimate of our natural self-propelled motion (Figure 1) $(39,40)$. However, passive artificial motion (e.g., experienced on a transport system) induces unnatural motion stimuli that lead to combinations of sensory signals judged impossible by our brain. Since each sensory signal could be interpreted as resulting from a different natural motion, our brain has to solve a problem of conflicting information, usually termed the sensory conflict (41-43).

\section{THEORIES ON MOTION SICKNESS}

The sensory mismatch theory $(2,44,45)$, the most widely accepted theory on motion sickness, states that conflict of motion stimuli alone is not sufficient to cause motion sickness. This conflict is only perceived as nauseogenic, if the present pattern of the rearranged sensory motion signals is at variance with what is expected from previous experience (2). A mathematical formulation of this theory defines the conflict vector as the difference between all sensed and all expected sensory information $(42,43,46)$.

The computation of this conflict vector can be simplified, if one only considers the difference between the subjective vertical and the vertical provided by the sensory signals, as proposed by the so-called subjective vertical conflict theory (47-50). On first consideration, the sensory mismatch theory and the subjective vertical conflict theory appear to be equivalent, but centering the computation of the conflict on the perception of verticality has

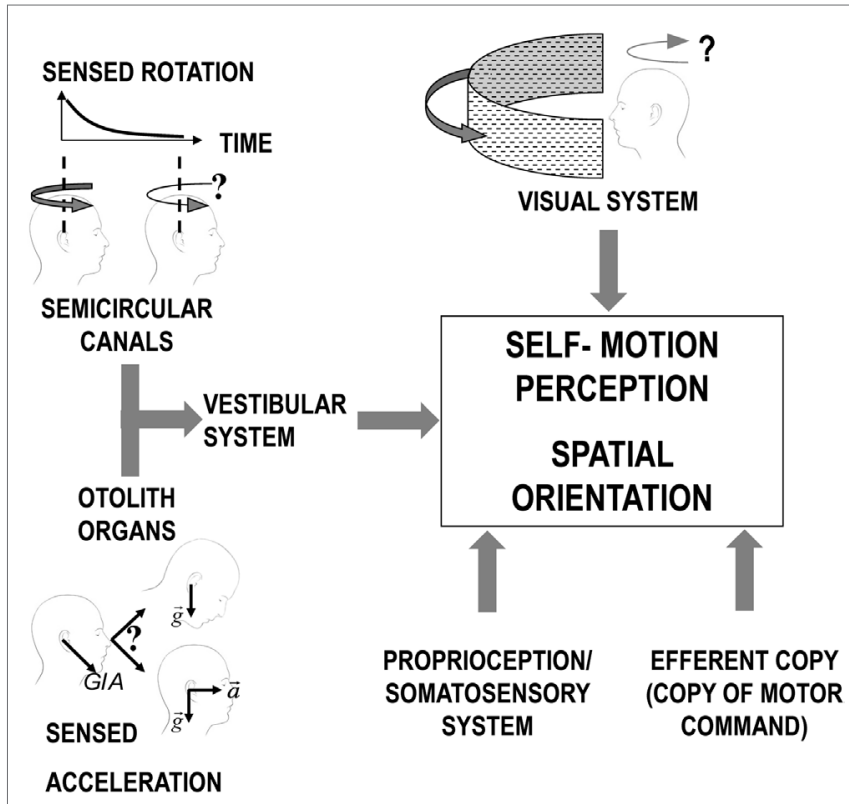

FIGURE 1 | Scheme of the different sensory systems contributing to the perception of self-motion. For the vestibular system, the otolith organs and the semicircular canals are represented separated to allow evidencing the inherent limitations of the signal from each sensor before central processing. Specifically, the semicircular canals signal in response to continuous rotation decline over time (solid black line decaying in the plot in the top/left corner) leading to a similar decrease in the sensation of self-rotation (as depicted by the change in the curve arrow above the two heads). The otolith organs, instead, perceive only the overall GIA vector (sum of gravity and inertial acceleration) and cannot, therefore, distinguish between a head tilt and linear translation.

significant impact on the definition of the nauseogenity of specific stimuli. For example, in the course of an angular on-vertical axis velocity step (i.e., a short acceleration phase followed by constant-velocity rotation), in an illuminated environment, the vestibular and optokinetic (rotatory information detected from visual input) signals are conflicting due to their different dynamics (vestibular: high-pass filter; optokinetic: low-pass filter). According to the classical sensory mismatch theory, this condition should cause motion sickness while it should not according to the subjective vertical conflict theory $(51,52)$. In reality, even a simple visual stimulus rotating around an Earth-vertical axis might induce motion sickness in susceptible subjects. This, however, does not disprove the subjective vertical conflict theory since, as explicitly stated by the authors (53), motion sickness in these situations is usually weak, has a considerably long latency and could be caused by a minor discrepancy between the rotation axis and true verticality. These discrepancies could be caused by a misperception of the center of rotation (52), a uncertainty in the sensory signals (54) or a minor distortion of the perceived vertical occurring when the head is held still for a prolonged time in a position differing from the exact upright $(55,56)$.

A theory based on the dynamic properties of the angular vestibuloocular reflex and their dependence on gravitoinertial vector orientation links the theoretical and the physiological framework 
(57-59). It states that the motion sickness is related to the difference between the yaw eigenvector of velocity storage (60), the mechanism responsible for integrating and coordinating signals for self-motion perception (61-64), and the actual vestibular velocity signal. Although specifically designed for head rotations, this theory often parallels the subjective vertical one as the velocity storage processes the sensed rotational velocity vector to align it with the gravitoinertial vector in order to generate a congruent integrated signal. Its yaw eigenvector, which drives such process by reinforcing the associated component of the rotational vector, is therefore close to the spatial vertical (65). The difference between such vector and the actual vestibular velocity signal is, therefore, identical to the conflict defined by the subjective vertical theory, but its computation can be advantageous when studying certain motion stimuli (e.g., cross-coupling - see The Cross-Coupled Stimulus).

A different computational approach to quantify the sensory mismatch has been presented in a series of work by Holly (66-69). The innovative aspect of this approach stays in that it describes the perceived motion evoked by nauseogenic stimuli on the basis of the law of physics without the need of including any specific physiological characteristic of the sensory system involved. This theory redefines the "conflict vector" splitting it in two $3 \mathrm{D}$ vectors, namely, a "twist factor," computing the difference between the expected and the perceived rotation, and a "stretch factor," computing the difference between the expected and the perceived translation. The results of the model simulations show that the "stretch factor" is the most relevant factor in causing motion sickness in the majority of the conditions (69). Its magnitude depends on the decomposition of the gravitoinertial acceleration in linear acceleration and gravity, confirming the dominant role of the correct perception of verticality (i.e., of gravity vector) in determining motion sickness. Therefore, it should not be a surprise that the simulations match the predictions of the subjective vertical conflict theory, although Holly's approach changes the focus from a mismatch of verticality into a mismatch of perceived translation.

A theory offering a radically different perspective has been proposed by Riccio and Stoffregen with the name of ecological theory of motion sickness $(70,71)$. It states that motion sickness occurs when a combination of motion stimuli trigger postural instability (71-73). In support of this theory, different studies have shown not only correlations of motion sickness with postural instability in nauseogenic environments (74-76) but also of susceptibility to motion sickness and postural sway in the absence of visual cues (77). Similar to the previously discussed ones, this theory proposes a tight connection between motion sickness and verticality perception. Yet, it deems the perturbations of postural stability as the cause of motion sickness genesis, moving the focus from a perceptual to a sensory-motor process (73). The main problem of this theory is, therefore, that it excludes from nauseogenity all the conditions where no active postural stabilization is required, although motion sickness have been observed also when lying or sitting, a setup often used in centrifugation experiments (78, 79). Moreover, the theory assumes that the perceptual process of upright is subordinated to its motor control counterpart, a phenomenon that, although supported by early studies $(71,73)$, is debated (80). The correlation suggested by the ecological theory of motion sickness may still provide advantages for describing situations focused on tasks related to postural stability in nauseogenic motion environments $(81,82)$.

Notice that we choose to present the different theories mentioned above without discussing the functional, physiological, or evolutionary reasons why certain motion profiles lead to motion sickness. Physiological and/or evolutionary reasons have been widely debated, in part also in connection with the abovementioned theories (83). Such discussion is, however, outside the scope of the current review.

\section{CLASSIFICATIONS OF MOTION SICKNESS}

From the earliest studies of motion sickness, classifications of the stimuli causing motion sickness have been proposed $(2,45,84)$. The proposed categorization separates the stimuli based on the source of the conflicting sensory signal or on whether the sensory conflict occurs between two actual signals or for the absence of an expected one. Although multiple examples have been provided (84), assigning a specific stimulus to a single group is not always univocal. This approach has been complementary to the theories discussed in the previous section, aimed at providing unifying explanations to clarify the mechanism of motion sickness, independently of the sensors involved.

Overall, at least two major categories of sensory conflict can be distinguished (45): (1) conflict between angular (semicircular canals) and linear (otolith organs) vestibular input and (2) conflict between visual and vestibular input. Detailed examples for the stimuli of each category have been presented in early work $(2,84)$. Currently, this classification proves to be useful for focusing on a specific nauseogenic stimulus, reproducing it in a laboratory environment and studying possible countermeasures for practical applications. Yet, a deeper understanding of why it causes motion sickness requires specific modeling provided by the theories discussed above. Once a specific nauseogenic stimulus has been selected or identified by its motion profile and sensory involvement, it is, however, often difficult to link it to the general framework of the theories. This problem is enhanced by the lack of quantitative assessments of motion sickness, which is often assessed subjectively alone with questionnaires $(19,59$, 85-88). This complicates comparing motion sickness generated by the different experimental paradigms. A comparative evaluation of the assessment methods is, however, outside the scope of the current review. This review focuses on vestibular-only motion sickness (i.e., category 1) with the aim to present both the characteristic of the most relevant nauseogenic stimuli in this category and the way in which they match the prediction of the above-mentioned theories.

\section{VESTIBULAR MOTION SICKNESS}

Vestibular-only motion sickness is being provoked when conflicts occur among different sensory signals of the vestibular system. These conflicts depend on how signals from the semicircular canals, providing transient head rotation velocity signal, and from otolith organs, detecting the gravitoinertial force vector, are 
integrated. The basic assumption is that gravity is the only know constant acceleration. Accordingly, segregation of the gravity vector from the inertial vector can be obtained by separation of the slow- and fast-changing component of the gravitoinertial acceleration with the first representing gravity and the second the inertial acceleration [frequency segregation hypothesis (89-91)]. This estimate of the direction of gravity, considered by the brain highly reliable, is also combined with the perceived head rotation derived from the semicircular canals $(49,92)$, and the agreement of both inputs is essential for a correct estimation of the head orientation in space (93-95). A perceived head rotation that does not match with a corresponding change in gravity direction leads to a sensory conflict that, if iterated, evokes motion sickness.

The classic example of this kind of conflict occurs when the semicircular canals perceive head rotations about an axis that is not aligned with the estimated gravity vector, but the latter does not change its orientation relative to the head accordingly. This happens, for instance, anytime we rotate the head about an off-Earth-vertical axis during an ongoing rotation about an Earth-vertical axis (Figure 2). It was first described by Ernst Mach in Versuch 2 of einer andern Reihe von Rotationsversuchen ${ }^{1}$ (96). The second rotation of the head immediately produces ein eigenthümliches Drehgefühl. ${ }^{2}$ This additional rotation of the head during ongoing rotation is called Coriolis/cross-coupled stimulus and its impact on the vestibular sensors is referred to as Coriolis/ cross-coupling effect (Figure 2 and section The Cross-Coupled Stimulus for details). Repetitive cross-coupling effects are disorienting and nauseogenic $(9,57)$. The subjective vertical conflict theory, Holly's physical laws of motion theory, and the velocity storage theory are especially useful to clarify motion sickness evoked by cross-coupled stimuli $(68,97)$.

Although cross-coupling stimuli are the most known form of vestibular-only conflict, other combinations of vestibular stimuli can elicit motion sickness, even when the canal-otolith conflict is not so explicit. A typical example is provided by linear oscillations, where the absence of rotation may lead to "forgetting" the role of semicircular canals. In these situations, it is likely that their missing activation causes motion sickness $(98,99)$. The following sections detail these major groups of stimuli inducing vestibular-only motion sickness and discuss their interpretation in the framework of the general motion sickness theories.

\section{The Cross-Coupled Stimulus}

The practical importance of this stimulus was first noted in aerospace medicine (100). When during a sharp turn of a plane, the pilot rotates his head about an axis that is not parallel to the axis of the turn, the resulting stimulus vector acting on the semicircular canals is perpendicular to the plane spanned by the two rotation axes (9). This phenomenon may occur in any curve around an Earth-vertical axis and, it is caused by the inherent decay of the response of semicircular canals to sustained rotations (Figures 1 and 2A). In fact, this decay leads to the computation

\footnotetext{
${ }^{1}$ Translation by Young, Henn \& Scherberger (Mach), 2001: another series of rotation experiments.

${ }^{2}$ Translation by Young, Henn \& Scherberger (Mach), 2001: strange sensation of turning.
}

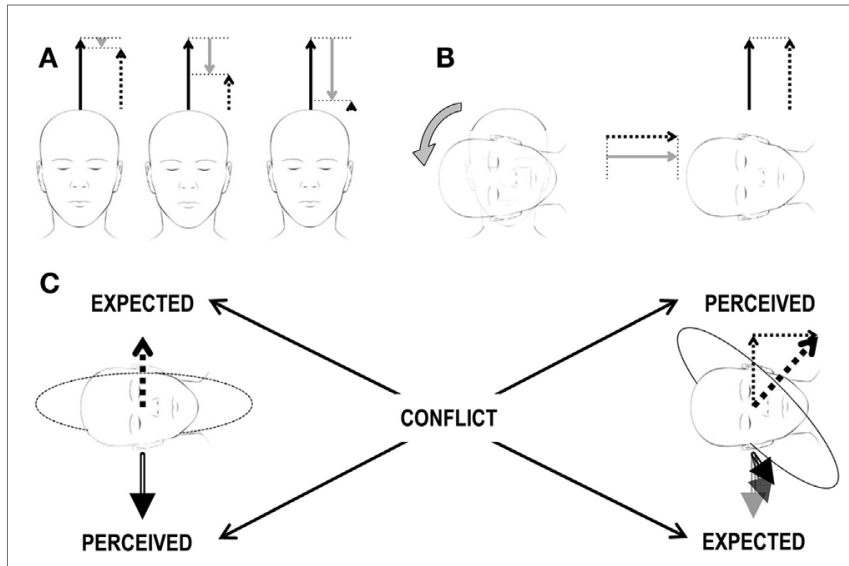

FIGURE 2 | Scheme of the Coriolis/cross-coupling stimulus and the induced sensory conflict. (A) Constant-velocity rotation. The three heads correspond to three subsequent time points during a constant-velocity rotation. The three arrows above each head correspond to the actual rotational velocity (solid black arrow), to the perceived velocity (dashed arrow), and to the amount of velocity decayed over time (gray arrow) due to the properties of the vestibular system. The sum of the solid black and gray arrows must always correspond to the dashed arrow. In the beginning (leftmost head), the subject has just been accelerated to the constant velocity. The perceived velocity (dashed arrow) is only slightly lower then the actual one (solid black arrow). As time passes (central and rightmost heads), the decayed velocity signal increases and the perceived velocity decreases. (B) Head tilt during constant-velocity rotation. Heads and arrows as in (A) after the tilt (rightmost head), no actual rotational velocity exists along the head vertical axis (no solid black arrow). However, as the decayed velocity is different from 0 , the absence of an actual velocity leads to a perceived velocity different from 0 (recall that sum of the solid black and gray arrows must always correspond to the dashed arrow, so in the absence of a black arrow, the dashed arrow is equal to the gray arrow). The actual velocity is now along the head interaural axis and is perceived by the semicircular canals. The perceived velocity has, therefore, now two orthogonal components. (C) Brain expectations and conflict. Leftmost head: the perceived gravity signal (double-line, black arrow below the head) is stable. Accordingly, any expected perceived rotational velocity (dashed arrow above the head) must be aligned with the gravity vector. Rightmost head: the perceived rotational velocity is tilted by $45^{\circ}$ (thick dashed arrow above the head) as it corresponds to the sum of the two perceived rotational velocity vectors in (B) (thin dashed arrows above the head). Accordingly, the gravity vector (double-line, black arrow below the head) should rotate in the head reference frame as the head changes orientation relative to gravity. Expected and perceived sensory signals do not match, leading to a sensory conflict.

of a false estimate of angular velocity. The changes of the velocity components occurring while the head is reoriented are, therefore, added to an incorrect starting point (Figure 2B), and the resulting perceived axis of rotation is incorrect, i.e., not aligned with the static gravity vector. Such combination of sensory inputs represents an impossible conflict as any expected perceived rotational velocity vector must be aligned with the gravity vector, if it is stable; otherwise, the gravity vector should rotate in the head reference frame as the head changes orientation relative to gravity (Figure 2C).

Coriolis/cross-coupling may lead to disorientation, motion sickness, and even aircraft casualties (9, 100, 101). Originally the cross-coupled stimulus was referred to as "Coriolis effect," because the effect was related to the linear movement of the head 
in a rotating plane (100); later, it was shown that the stimulus can be calculated by the cross-product of the active and passive rotations acting on the semicircular canals $(9,57,102-104)$ and was, therefore, referred to as cross-coupling stimulus. In microgravity, the cross-coupled stimulus has the same effect on the semicircular canals, but the disorienting and nauseogenic effect is reduced or even missing (105). This was demonstrated during the Skylab mission M131 $(106,107)$ where astronauts were capable of repetitively rolling their head while rotating without getting motion sick, while they perceived the same stimulus as nauseogenic on Earth. This finding provides direct evidence that the mechanism underling motion sickness induced by crosscoupling depends on the orientation of the rotation vector with respect to gravity, as predicted by the subjective vertical and the velocity storage theories. The reduced sensitivity to Coriolis/ cross-coupling in microgravity implies that in absence of gravity (or any space fixed gravitoinertial force), the illusory rotation signal is not perceived as conflicting as it cannot be integrated and compared with the gravity signal. The conflict is, therefore, missing.

The disorienting and nauseogenic effects of cross-coupled head-roll stimuli depend on the acceleration (direction and magnitude) of the first rotation. If the head is tilted around a roll axis during the acceleration phase of Earth-vertical yaw rotation, the cross-coupling effect is smaller than during the deceleration phase (9). During the acceleration phase, the summed vector of yaw acceleration and cross-coupled pitch acceleration is much closer to gravity than the summed vector of yaw deceleration and cross-coupled pitch acceleration. Hence, the canal-otolith conflict is the largest during yaw deceleration, smallest during yaw acceleration, and in-between during constant-velocity yaw.

Whether head tilts during ongoing yaw rotation are active, e.g., by verbally instructed head roll (9), or passive, e.g., by whole-body tilts on a 3D motorized turntable (108), seems equally disorienting. However, if both cross-coupled rotations are actively performed as part of a natural movement, e.g., during locomotion (109), no disorientation or motion sickness occurs. Guedry and Benson provided a conclusive explanation for this fact (9): during natural movements, cross-coupled rotations always occur during the acceleration phase of a yaw rotation, which minimizes the conflict with the otolith signal; however, cross-coupled rotations during constant-velocity yaw or during deceleration from constant-velocity yaw are not part of natural movement repertoire.

\section{Cross-Coupled Rotations, Motion Sickness Theories, and Velocity Storage}

All of the above-mentioned theories are able to explain why motion sickness is induced by cross-coupling stimuli. The sensory conflict is indeed evident as semicircular canals signals a rotation axis in conflict with the one suggested by changes gravitoinertial force. By describing how the stimulus should be perceived by the brain according to the law of physics, Holly specifically showed that both stretch and twist factors are different from 0 (68). The direct involvement of the gravity direction in the conflict confirms the central role of verticality perception and matches, therefore, the prediction of the subjective vertical theory (97). Similarly, it was demonstrated that the velocity storage mechanism (60) plays an important role in making the cross-coupled stimulus nauseogenic (58). It is worth to note that in this condition, the subjective vertical theory (47) seems to be experimentally indistinguishable from the velocity storage theory. During a constantvelocity rotation, the yaw axis eigenvector of velocity storage is identical to the spatial vertical, and therefore, the conflict vector computed according to the two theories between such vector and the vestibular velocity signal evoked by the head tilt is identical (see Theories on Motion Sickness for details). However, the latter hypothesis makes the precise prediction that the cerebellar nodulus and uvula, which control the dynamics and 3D kinematics of velocity storage (110-112), are the main central structures involved in motion sickness.

The importance of the time constant of velocity storage in determining the motion sickness sensitivity during paradigms with repetitive cross-coupled stimuli is remarkable $(57,80)$. During constant-velocity yaw rotation, subjects with short time constants were able to make more head-roll movements before reaching full motion sickness than subjects with long time constants. This can also explain why subjects can be habituated to cross-coupled stimuli (113-115), which is most likely due to the reduction in the velocity storage time constant with repetition $(78,116,117)$. The decrease in motion sickness susceptibility by baclofen probably works via the same mechanism. Interestingly, the relation between velocity storage time constant and motion sickness appears to have a general validity, as individuals with long time constants are more prone to motion sickness (118, 119), while shortening the time constant pharmacologically or by habituation reduces motion sickness sensitivity (113, 120-122). The role of the velocity storage in determining the nauseogenity of the cross-coupling stimuli can also explain the reduced motion sickness caused by such stimuli in weightless condition (i.e., astronauts or during parabolic flight). The absence of the gravity load on the otolith organs, indeed, deactivates the velocity storage removing, therefore, the source of the conflict $(80,123)$.

The link between motion sickness and velocity storage has also relevance for the assessment of nauseogenic stimuli through vestibular responses. In the absence of ocular motor deficits, the 3D angular velocity vector of the eyes in total darkness closely reflects the $3 \mathrm{D}$ central vestibular velocity signal. This signal represents the sum of the afferent vestibular signal and a partly integrated version of this signal, i.e., the output of the velocity storage (60). While the direct vestibular signal is three-dimensionally isotropic, the velocity storage mechanism in humans acts predominantly in the yaw plane (124-126). Recording eye movements in three dimensions (horizontal, vertical, and torsional) lends itself to study the impact of cross-coupled stimuli on the central vestibular system by analyzing the $3 \mathrm{D}$ disparity between head and eye movements $(57,108)$. Moreover, eye movement parameters, such as gain and time constant of vestibular nystagmus, can be related to measures of motion sickness and to monitor the effectiveness of habituation protocols $(78,79,118,121,127)$.

\section{Linear Oscillations}

The role of linear oscillation in determining motion sickness was first studied in relation to seasickness, i.e., motion sickness 
induced by the oscillatory motion of a boat at sea. While some studies were done in real ships and hovercraft $(128,129)$, others were conducted in simulated condition, isolating the linear from the angular component of the motion (130-132). All these experiments agreed that vertical linear oscillation at low frequency is the most important stimulus in causing seasickness (129). Specifically, any oscillation at frequencies between 0.1 and $0.5 \mathrm{~Hz}$ was found to be nauseogenic, with a peak around $0.16 \mathrm{~Hz}(129$, 131-133). Within a given frequency, motion sickness increases monotonically with increasing acceleration (131, 132). More recent studies showed that similar responses are evoked by linear horizontal oscillations in both lateral and fore-aft directions (99, 134-141). By comparing fore and aft and vertical acceleration of similar magnitudes and frequencies, Golding and colleagues found that the first causes stronger motion sickness than their vertical counterpart (99).

The motion sickness induced by linear oscillations may apparently contrast with the sensory conflict theory, since they cause variations of a single sensory signal. Moreover, since high frequency oscillation do not apparently cause disturbances (142), it is clear that not all oscillatory movements cause motion sickness. Physical motion at frequency higher than $1 \mathrm{~Hz}$ not only does induce motion sickness but can also be beneficial if added to lowfrequency oscillation as it reduces the reliability of the conflicting sensory input (143). The picture becomes clearer when recalling that the conflicts of the sensory mismatch theory are defined as a discrepancy between the brain expectation and the actual arrangement of sensory inputs $(2,42-46)$. To understand how sensory inputs are processed during linear oscillation, one must take into account that the otolith signal is normally low pass filtered to separate the gravity vector, expected to undergo relatively slow variations in direction and the inertial component of the sensed gravitoinertial vector (frequency segregation hypothesis) (89-91). Accelerations during linear oscillations below a specific frequency are, therefore, added to gravity as the brain fails to identify them correctly and perceives an unexpected change of the gravity vector, unmatched by other sensory signals. The frequency segregation processing is also at the base of the computation of the error vector according to the subjective vertical theory (47-50). To reproduce the differences between horizontal and vertical oscillations, however, the initial theory was extended including an additional internal conflict signal, specific for horizontal linear accelerations [subjective vertical-horizontal theory (SVH)] (144). The SVH theory has been successfully used to predict motion sickness on different vessels at sea (145). Successful prediction of the nauseogenity of linear oscillation at specific frequency can be obtained by applying the theories of Holly (69), with the advantage that the stretch factor represents a linear displacement error resulting from multisensory computation and requires, therefore, no differentiation between vertical and horizontal acceleration conflicts.

\section{Combined Linear and Angular Oscillation}

In real condition (e.g., for a vessel at sea or on a bus on a curvy road), it is unlikely that the oscillations are limited to a singular dimension. For example, a small boat hit by a sequence of waves oscillates vertically (heave) at the frequency of the coming waves, but it also shows pitch and roll oscillatory motion that depends on its structural characteristic. Since the early studies on motion sickness at sea $(3,128,129)$ were conducted on large ships, providing weak pitch-roll stimulation, it was concluded that heave was the sole nauseogenic stimuli at sea. However, to investigate the reasons behind the widely spread belief that travels on small boats are more provocative than large one (146), simulation studies have been specifically designed to investigate whether combination of angular and linear oscillations are more provocative than linear oscillations alone (147). Although earlier studies found no differences $(132,148)$ when combinations of heave and pitch and roll oscillations were used, an increase in motion sickness was observed in a majority of recent studies $(134,135,139,147,149)$. Overall, the provocativeness of combination of angular and linear oscillations depends on the specific combination of acceleration and frequency content of the two stimuli $(135,147)$.

\section{EXAMPLE OF VESTIBULAR MOTION SICKNESS IN EVERYDAY LIFE}

\section{Motion Sickness in Tilting Trains}

Tilting the car bodies of trains compensates for the centripetal acceleration during turns by bringing the vertical axes of the cars closer to the gravitoinertial force vector. As a result, the trains can run faster and the lateral thrusts of centrifugal force on the passengers during turns are decreased. Unfortunately, many passengers in tilting trains develop symptoms of motion sickness, a major problem of modern traffic $(4,150)$. A detailed understanding of the mechanism underlying motion sickness in tilting trains is essential because only such knowledge allows developing technical solutions for the problem (135).

Tilting trains provide an ideal example of how a real, everyday motion condition that induces motion sickness comprises multiple nauseogenic stimuli. Accurate recording of the linear and angular motion of the cars showed that at least three could be the cause of motion sickness in the train: the centrifugal acceleration mentioned above, the jitters of the car, which shows peaks at 0.5 and $1.7 \mathrm{~Hz}$ both in roll rotation and lateral translation (88), and the tilt of train itself, as it provides a typical cross-coupled stimulus with roll movements during both the acceleration and deceleration phases of yaw rotation (88). Various studies showed the nauseogenic role of the lateral linear acceleration in actual and simulated conditions, in particular if combined with roll tilts $(99,135,137,139,150,151)$. Accordingly, motion sickness of passengers in tilting trains can be reduced by decreasing the angle of the compensatory roll tilt $(4,88,152)$. The usefulness of this approach is limited because the velocity of trains on curves has to decrease, which negates the purpose of the tilt. With respect to the jitter, while $1.7 \mathrm{~Hz}$ oscillations are way above the nauseogenic range, $0.5 \mathrm{~Hz}$ is likely causing disturbances (see Linear Oscillation). Finally, cross-coupling (see Cross-coupling) is one of the most powerful nauseogenic stimuli; however, whether the relatively low yaw velocities around $4 \%$, which are typical on curved tracks, are adequate to produce cross-coupling motion sickness is not known. Interestingly, it has been observed that if subjects' heads were tilted during lateral acceleration, they had 
strong motion sickness, but if the head roll was initiated before the lateral acceleration, there was no motion sickness (153). Similarly, by comparing different tilting systems, it has been recently showed that adequate synchronization of roll tilt with changes of yaw velocity on curves, however, eliminates motion sickness (88). These findings clearly suggest that the phasing between the change of yaw velocity and the change of roll position seems to be a decisive factor and confirm that cross-coupling also plays a role in the nauseogenity of tilting trains. Overall, these studies, by analyzing different aspects of the train motion stimuli, underline the agreement between the nauseogenity of an actual motion and the prediction of the theories presented above. The sustained lateral acceleration due to centripetal force causes a tilt of the perceived direction of gravity that depends mainly on the radius of the curve (as the angular velocity of the train is relatively small). If in these conditions, the perceived rotation is further misaligned by a delayed roll tilt, the resulting cross-coupling can be significantly nauseogenic and the amount of conflict can be computed according to the misalignment in the velocity storage vectors, the discrepancy in the perceived and expected subjective vertical, or the stretch and twist factors.

\section{Motion Sickness in Cars and Public Road Transport}

Motion sickness in road vehicle is a very common experience, with high incidence of vomiting, particularly in young passengers between the age of 2 and 12 years $(154,155)$. By correlating motion sickness with car motions, multiple studies evidenced that the relevant nauseogenic stimuli in road transport are the low-frequency $(<0.5 \mathrm{~Hz})$ horizontal linear oscillations (155-157). Relative contributions of fore-aft and lateral accelerations seem to be similar as well as similar are their acceleration spectra in the frequency range relevant for inducing sickness $(0.1-05 \mathrm{~Hz})(156)$. The vertical component of linear motion instead has relevant magnitude only between 1 and $2 \mathrm{~Hz}$ (142) and, according to the studies on linear oscillation (see Linear Oscillations), does not appear to cause discomfort $(99,158)$. Hence, motion sickness occurrence in cars and public road transport seems to be mainly influenced by the quality of the driver and the condition of the road (e.g., curvy cross-country versus highway), both factors determining lateral oscillations. The mechanical characteristics of the cars (e.g., active suspension), which control the amount

\section{REFERENCES}

1. Irwin JA. The pathology of sea-sickness. The Lancet (1881) 118:907-9. doi:10.1016/S0140-6736(02)38129-7

2. Reason JT, Brand JJ. Motion Sickness. London: Academic Press (1975).

3. Lawther A, Griffin MJ. A survey of the occurrence of motion sickness amongst passengers at sea. Aviat Space Environ Med (1988) 59(5):399-406.

4. Forstberg J, Andersson E, Ledin T. Influence of different conditions for tilt compensation on symptoms of motion sickness in tilting trains. Brain Res Bull (1998) 47(5):525-35. doi:10.1016/S0361-9230(98)00097-5

5. Reschke MF, Bloomberg JJ, Harm DL, Paloski WH, Layne C, McDonald V. Posture, locomotion, spatial orientation, and motion sickness as a function of space flight. Brain Res Brain Res Rev (1998) 28(1-2):102-17. doi:10.1016/ S0165-0173(98)00031-9

6. Lackner JR, Dizio P. Space motion sickness. Exp Brain Res (2006) 175(3):377-99. doi:10.1007/s00221-006-0697-y of vertical oscillations, were shown not to play a determinant role $(142,158,159)$. The variation of motion sickness with seat position, increasing from the front to the back of the vehicles, can also be explained by the corresponding increase in the magnitude of lateral oscillations (158). The known fact that drivers rarely become motion sick may be due to the driver's prediction of low-frequency horizontal accelerations as they depend on the driver's behavior $(158,160,161)$. This prediction may allow engaging various compensatory actions, such as tilting the head to align with the tilted gravitoinertia, therefore minimizing the lateral oscillations, a behavior often observed in the drivers (161). Accordingly, motion sickness in rally co-drivers varies considerably as a function of the specific activity, which may involve different levels of interaction with the driving and the environment (162). A recent study, asking the passenger to actively align the head to the gravitointertia, mimicking therefore the driver's behavior, showed a significant reduction in motion sickness with respect to normal seating with the head upright $(163,164)$. These considerations are also of great importance for the design of selfdriving cars. As suggested by a recent survey on the preferred activity to be done in a self-driving vehicle (165), the benefit of such emerging technology stays in the freedom to disengage from the driving activity and the surrounding environment, therefore increasing the sensory conflict. Any activity causing a strong sensory conflict, however, will be unbearable for motion sickness sensitive individuals and will be partially hindered in more robust ones, reducing the potentially positive impact of this novel technology on the society (166).

\section{AUTHOR CONTRIBUTIONS}

The authors wrote the review, collected the references by literature search, and edited the final version.

\section{FUNDING}

Swiss National Science Foundation, Bern, Switzerland; Betty and David Koetser Foundation for Brain Research, Zurich, Switzerland; Center of Integrative Human Physiology, University of Zurich, Zurich, Switzerland; Bonizzi-Theler-Foundation, Zurich, Switzerland; and Dr. Dabbous Foundation, Zurich, Switzerland.

7. Turner M, Griffin MJ. Motion sickness in public road transport: the relative importance of motion, vision and individual differences. Br J Psychol (1999) 90(Pt 4):519-30. doi:10.1348/000712699161594

8. Walker AD, Muth ER, Switzer FS, Hoover A. Head movements and simulator sickness generated by a virtual environment. Aviat Space Environ Med (2010) 81(10):929-34. doi:10.3357/ASEM.2735.2010

9. Guedry FE Jr, Benson AJ. Coriolis cross-coupling effects: disorienting and nauseogenic or not? Aviat Space Environ Med (1978) 49(1 Pt 1):29-35.

10. DiZio P, Lackner JR, Evanoff JN. The influence of gravitoinertial force level on oculomotor and perceptual responses to coriolis, cross-coupling stimulation. Aviat Space Environ Med (1987) 58(9 Pt 2):A218-23.

11. Miller EF II, Graybiel A. Motion sickness produced by head movement as a function of rotational velocity. Aerosp Med (1970) 41(10):1180-4.

12. Murdin L, Golding J, Bronstein A. Managing motion sickness. BMJ (2011) 343:d7430. doi:10.1136/bmj.d7430

13. Money KE. Motion sickness. Physiol Rev (1970) 50(1):1-39. 
14. Lentz JM, Guedry FE Jr. Motion sickness susceptibility: a retrospective comparison of laboratory tests. Aviat Space Environ Med (1978) 49(11):1281-8. doi:10.1016/0022-460x(80)90337-5

15. Golding JF. Motion sickness susceptibility. Auton Neurosci (2006) 129(12):67-76. doi:10.1016/j.autneu.2006.07.019

16. Paillard AC, Quarck G, Paolino F, Denise P, Paolino M, Golding JF, et al. Motion sickness susceptibility in healthy subjects and vestibular patients: effects of gender, age and trait-anxiety. J Vestib Res (2013) 23(4-5):203-9. doi:10.3233/VES-130501

17. Cheung BS, Money KE, Jacobs I. Motion sickness susceptibility and aerobic fitness: a longitudinal study. Aviat Space Environ Med (1990) 61(3):201-4.

18. Golding JF, Kadzere P, Gresty MA. Motion sickness susceptibility fluctuates through the menstrual cycle. Aviat Space Environ Med (2005) 76(10):970-3.

19. Golding JF. Motion sickness susceptibility questionnaire revised and its relationship to other forms of sickness. Brain Res Bull (1998) 47(5):507-16. doi:10.1016/S0361-9230(98)00091-4

20. Reavley CM, Golding JF, Cherkas LF, Spector TD, MacGregor AJ. Genetic influences on motion sickness susceptibility in adult women: a classical twin study. Aviat Space Environ Med (2006) 77(11):1148-52.

21. Golding JF, Gresty MA. Pathophysiology and treatment of motion sickness. Curr Opin Neurol (2015) 28(1):83-8. doi:10.1097/WCO.0000000000000163

22. Murdin L, Chamberlain F, Cheema S, Arshad Q, Gresty MA, Golding JF, et al. Motion sickness in migraine and vestibular disorders. J Neurol Neurosurg Psychiatry (2014) 86(5):585-7. doi:10.1136/jnnp-2014-308331

23. Kuritzky A, Ziegler DK, Hassanein R. Vertigo, motion sickness and migraine. Headache (1981) 21(5):227-31. doi:10.1111/j.1526-4610.1981.hed2105227.x

24. Barabas G, Matthews WS, Ferrari M. Childhood migraine and motion sickness. Pediatrics (1983) 72(2):188-90.

25. Jeong SH, Oh SY, Kim HJ, Koo JW, Kim JS. Vestibular dysfunction in migraine: effects of associated vertigo and motion sickness. J Neurol (2010) 257(6):905-12. doi:10.1007/s00415-009-5435-5

26. Cutrer FM, Baloh RW. Migraine-associated dizziness. Headache (1992) 32(6):300-4. doi:10.1111/j.1526-4610.1992.hed3206300.x

27. Baloh RW. Neurotology of migraine. Headache (1997) 37(10):615-21. doi:10.1046/j.1526-4610.1997.3710615.x

28. Murdin L, Davies RA, Bronstein AM. Vertigo as a migraine trigger. Neurology (2009) 73(8):638-42. doi:10.1212/WNL.0b013e3181b38a04

29. Cuomo-Granston A, Drummond PD. Migraine and motion sickness: what is the link? Prog Neurobiol (2010) 91(4):300-12. doi:10.1016/j. pneurobio.2010.04.001

30. Graybiel A. Vestibular sickness and some of its implications for space flight. In: Field WS, Alfords BR, editors. Neurological Aspects of Auditory and Vestibular Disorders. Springfield, Ill: CC Thomas (1963).

31. Kennedy RS, Graybiel A, McDonough RC, Beckwith FD. Symptomatology under storm conditions in the North Atlantic in control subjects and in persons with bilateral labyrinthine defects. Acta Otolaryngol (1968) 66(6):533-40. doi:10.3109/00016486809126317

32. Cheung BS, Howard IP, Money KE. Visually-induced sickness in normal and bilaterally labyrinthine-defective subjects. Aviat Space Environ Med (1991) 62(6):527-31.

33. Dichgans J, Brandt T. Optokinetic motion sickness and pseudo-Coriolis effects induced by moving visual stimuli. Acta Otolaryngol (1973) 76(5):33948. doi:10.3109/00016487309121519

34. Bos JE, de Vries SC, van Emmerik ML, Groen EL. The effect of internal and external fields of view on visually induced motion sickness. Appl Ergon (2010) 41(4):516-21. doi:10.1016/j.apergo.2009.11.007

35. Diels C, Howarth PA. Frequency characteristics of visually induced motion sickness. Hum Factors (2013) 55(3):595-604. doi:10.1177/0018720812469046

36. Angelaki DE, Cullen KE. Vestibular system: the many facets of a multimodal sense. Annu Rev Neurosci (2008) 31:125-50. doi:10.1146/annurev. neuro.31.060407.125555

37. Leigh RJ, Zee DS. The Neurology of Eye Movements. 4th ed. New York: Oxford University Press (2006).

38. Einstein A. Über das relativitätsprinzip und die aus demselben gezogenen folgerungen. Jahrb Radioakt (1908) 4:411-62.

39. Fetsch CR, Turner AH, DeAngelis GC, Angelaki DE. Dynamic reweighting of visual and vestibular cues during self-motion perception. J Neurosci (2009) 29(49):15601-12. doi:10.1523/JNEUROSCI.2574-09.2009
40. Knill DC, Pouget A. The Bayesian brain: the role of uncertainty in neural coding and computation. Trends Neurosci (2004) 27(12):712-9. doi:10.1016/j. tins.2004.10.007

41. Brandt T, Daroff RB. The multisensory physiological and pathological vertigo syndromes. Ann Neurol (1980) 7(3):195-203. doi:10.1002/ana.410070302

42. Oman CM. A heuristic mathematical model for the dynamics of sensory conflict and motion sickness. Acta Otolaryngol Suppl (1982) 392:1-44.

43. Oman CM. Motion sickness: a synthesis and evaluation of the sensory conflict theory. Can J Physiol Pharmacol (1990) 68(2):294-303. doi:10.1139/y90-044

44. Reason JT. Motion sickness: a special case of sensory rearrangement. Adv Sci (1970) 26(130):386-93.

45. Reason JT. Motion sickness adaptation: a neural mismatch model. $J R$ Soc Med (1978) 71(11):819-29.

46. Brandt T. Vertigo: Its Multisensory Syndromes. London: Springer (2003).

47. Bles W, Bos JE, de Graaf B, Groen E, Wertheim AH. Motion sickness: only one provocative conflict? Brain Res Bull (1998) 47(5):481-7. doi:10.1016/ S0361-9230(98)00115-4

48. Bos JE, Bles W. Modelling motion sickness and subjective vertical mismatch detailed for vertical motions. Brain Res Bull (1998) 47(5):537-42. doi:10.1016/ S0361-9230(98)00088-4

49. Bos JE, Bles W. Theoretical considerations on canal-otolith interaction and an observer model. Biol Cybern (2002) 86(3):191-207. doi:10.1007/ s00422-001-0289-7

50. Groen E, Bos J, de Graaf B, Bles W. Otolith signal processing and motion sickness. Ann N Y Acad Sci (1999) 871:406-9. doi:10.1111/j.1749-6632.1999. tb09205.x

51. Bles W, Bos JE, Kruit H. Motion sickness. Curr Opin Neurol (2000) 13(1):19-25. doi:10.1097/00019052-200002000-00005

52. Bos JE, Bles W. Motion sickness induced by optokinetic drums. Aviat Space Environ Med (2004) 75(2):172-4.

53. Bos J, Bles W, Groen E. A theory on visually induced motion sickness. Health Saf Aspects Visual Disp (2008) 29(2):47-57. doi:10.1016/j.displa.2007.09.002

54. Tarnutzer AA, Bockisch C, Straumann D, Olasagasti I. Gravity dependence of subjective visual vertical variability. J Neurophysiol (2009) 102(3):1657-71. doi:10.1152/jn.00007.2008

55. Tarnutzer AA, Bockisch CJ, Straumann D, Marti S, Bertolini G. Static roll-tilt over 5 minutes locally distorts the internal estimate of direction of gravity. J Neurophysiol (2014) 112(11):2672-9. doi:10.1152/jn.00540.2014

56. Tarnutzer AA, Bertolini G, Bockisch CJ, Straumann D, Marti S. Modulation of internal estimates of gravity during and after prolonged roll-tilts. PLoS One (2013) 8(10):e78079. doi:10.1371/journal.pone.0078079

57. Dai M, Kunin M, Raphan T, Cohen B. The relation of motion sickness to the spatial-temporal properties of velocity storage. Exp Brain Res (2003) 151(2):173-89. doi:10.1007/s00221-003-1479-4

58. Cohen B, Dai M, Raphan T. The critical role of velocity storage in production of motion sickness. Ann N Y Acad Sci (2003) 1004:359-76. doi:10.1196/ annals.1303.034

59. Dai M, Sofroniou S, Kunin M, Raphan T, Cohen B. Motion sickness induced by off-vertical axis rotation (OVAR). Exp Brain Res (2010) 204(2):207-22. doi:10.1007/s00221-010-2305-4

60. Raphan T, Matsuo V, Cohen B. Velocity storage in the vestibulo-ocular reflex $\operatorname{arc}$ (VOR). Exp Brain Res (1979) 35(2):229-48. doi:10.1007/BF00236613

61. Laurens J, Angelaki DE. The functional significance of velocity storage and its dependence on gravity. Exp Brain Res (2011) 210(3-4):407-22. doi:10.1007/ s00221-011-2568-4

62. Bertolini G, Ramat S, Laurens J, Bockisch CJ, Marti S, Straumann D, et al. Velocity storage contribution to vestibular self-motion perception in healthy human subjects. J Neurophysiol (2011) 105(1):209-23. doi:10.1152/ jn. 00154.2010

63. Bertolini G, Ramat S, Bockisch CJ, Marti S, Straumann D, Palla A. Is vestibular self-motion perception controlled by the velocity storage? Insights from patients with chronic degeneration of the vestibulo-cerebellum. PLoS One (2012) 7(6):e36763. doi:10.1371/journal.pone.0036763

64. Okada T, Grunfeld E, Shallo-Hoffmann J, Bronstein AM. Vestibular perception of angular velocity in normal subjects and in patients with congenital nystagmus. Brain (1999) 122(Pt 7):1293-303. doi:10.1093/brain/122.7.1293

65. Raphan T, Dai M, Cohen B. Spatial orientation of the vestibular system. Ann N Y Acad Sci (1992) 656:140-57. doi:10.1111/j.1749-6632.1992.tb25205.x 
66. Holly JE. Baselines for three-dimensional perception of combined linear and angular self-motion with changing rotational axis. J Vestib Res (2000) 10(4-5):163-78.

67. Holly JE. Perceptual disturbances predicted in zero-g through threedimensional modeling. J Vestib Res (2003) 13(4-6):173-86.

68. Holly JE. Vestibular coriolis effect differences modeled with threedimensional linear-angular interactions. J Vestib Res (2004) 14(6):443-60.

69. Holly JE, Harmon SM. Sensory conflict compared in microgravity, artificial gravity, motion sickness, and vestibular disorders. J Vestib Res (2012) 22(2):81-94. doi:10.3233/VES-2012-0441

70. Riccio DC, Stoffregen TA. An ecological theory of motion sickness and postural instability. Ecol Psychol (1991) 3:195-240. doi:10.1207/ s15326969eco0303_2

71. Stoffregen TA, Riccio GE. An ecological theory of orientation and the vestibular system. Psychol Rev (1988) 95(1):3-14. doi:10.1037/0033-295X.95.1.3

72. Smart LJ Jr, Pagulayan RJ, Stoffregen TA. Self-induced motion sickness in unperturbed stance. Brain Res Bull (1998) 47(5):449-57. doi:10.1016/ S0361-9230(98)00103-8

73. Stoffregen TA, Smart LJ Jr. Postural instability precedes motion sickness. Brain Res Bull (1998) 47(5):437-48. doi:10.1016/S0361-9230(98)00102-6

74. Varlet M, Bardy BG, Chem FC, Alcantara C, Stoffregen TA. Coupling of postural activity with motion of a ship at sea. Exp Brain Res (2015) 233(5):1607-16. doi:10.1007/s00221-015-4235-7

75. Tal D, Bar R, Nachum Z, Gil A, Shupak A. Postural dynamics and habituation to seasickness. Neurosci Lett (2010) 479(2):134-7. doi:10.1016/j. neulet.2010.05.044

76. Yokota Y, Aoki M, Mizuta K, Ito Y, Isu N. Motion sickness susceptibility associated with visually induced postural instability and cardiac autonomic responses in healthy subjects. Acta Otolaryngol (2005) 125(3):280-5. doi:10.1080/00016480510003192

77. Owen N, Leadbetter AG, Yardley L. Relationship between postural control and motion sickness in healthy subjects. Brain Res Bull (1998) 47(5):471-4. doi:10.1016/S0361-9230(98)00101-4

78. Garrick-Bethell I, Jarchow T, Hecht H, Young LR. Vestibular adaptation to centrifugation does not transfer across planes of head rotation. J Vestib Res (2008) 18(1):25-37.

79. Young LR, Sienko KH, Lyne LE, Hecht H, Natapoff A. Adaptation of the vestibulo-ocular reflex, subjective tilt, and motion sickness to head movements during short-radius centrifugation. J Vestib Res (2003) 13(2-3):65-77.

80. Lackner JR. Motion sickness: more than nausea and vomiting. Exp Brain Res (2014) 232(8):2493-510. doi:10.1007/s00221-014-4008-8

81. Chen FC, Stoffregen TA. Specificity of postural sway to the demands of a precision task at sea. J Exp Psychol Appl (2012) 18(2):203-12. doi:10.1037/ a0026661

82. Varlet M, Stoffregen TA, Chen FC, Alcantara C, Marin L, Bardy BG. Just the sight of you: postural effects of interpersonal visual contact at sea. J Exp Psychol Hum (2014) 40(6):2310-8. doi:10.1037/a0038197

83. Oman CM. Are evolutionary hypotheses for motion sickness "just-so" stories? J Vestib Res (2012) 22(2):117-27. doi:10.3233/VES-2011-0432

84. Reason J. Motion sickness: some theoretical and practical considerations. Appl Ergon (1978) 9(3):163-7. doi:10.1016/0003-6870(78)90008-X

85. Gianaros PJ, Muth ER, Mordkoff JT, Levine ME, Stern RM. A questionnaire for the assessment of the multiple dimensions of motion sickness. Aviat Space Environ Med (2001) 72(2):115-9.

86. Graybiel A, Wood CD, Miller EF, Cramer DB. Diagnostic criteria for grading the severity of acute motion sickness. Aerosp Med (1968) 39(5):453-5.

87. Hecht H, Kavelaars J, Cheung CC, Young LR. Orientation illusions and heart-rate changes during short-radius centrifugation. J Vestib Res (2001) 11(2):115-27.

88. Cohen B, Dai M, Ogorodnikov D, Laurens J, Raphan T, Muller P, et al. Motion sickness on tilting trains. FASEB J (2011) 25(11):3765-74. doi:10.1096/ f. 11-184887

89. Mayne R. A systems concept of the vestibular organs. In: Kornhuber H, editor. Handbook of Sensory Physiology Vestibular System Psychophysics, Applied Aspects and General Interpretations. VI. Berlin: Springer-Verlag (1974). p. 493-580.
90. Zupan LH, Peterka RJ, Merfeld DM. Neural processing of gravitoinertial cues in humans. I. Influence of the semicircular canals following post-rotatory tilt. J Neurophysiol (2000) 84(4):2001-15.

91. Correia Gracio BJ, de Winkel KN, Groen EL, Wentink M, Bos JE. The time constant of the somatogravic illusion. Exp Brain Res (2013) 224(3):313-21. doi:10.1007/s00221-012-3313-3

92. Merfeld DM, Zupan L, Peterka RJ. Humans use internal models to estimate gravity and linear acceleration. Nature (1999) 398(6728):615-8. doi:10.1038/19303

93. Bertolini G, Wicki A, Baumann CR, Straumann D, Palla A. Impaired tilt perception in Parkinson's disease: a central vestibular integration failure. PLoS One (2015) 10(4):e0124253. doi:10.1371/journal.pone.0124253

94. Merfeld DM, Park S, Gianna-Poulin C, Black FO, Wood S. Vestibular perception and action employ qualitatively different mechanisms. I. Frequency response of VOR and perceptual responses during translation and tilt. J Neurophysiol (2005) 94(1):186-98. doi:10.1152/jn.00904.2004

95. Merfeld DM, Park S, Gianna-Poulin C, Black FO, Wood S. Vestibular perception and action employ qualitatively different mechanisms. II. VOR and perceptual responses during combined Tilt\&Translation. J Neurophysiol (2005) 94(1):199-205. doi:10.1152/jn.00905.2004

96. Mach E. Grundlinien der Lehre von den Bewegungsempfindungen. Leipzig: Engelmann (1875).

97. Bles W. Coriolis effects and motion sickness modelling. Brain Res Bull (1998) 47(5):543-9. doi:10.1016/S0361-9230(98)00089-6

98. Golding JF, Kerguelen M. A comparison of the nauseogenic potential of low-frequency vertical versus horizontal linear oscillation. Aviat Space Environ Med (1992) 63(6):491-7.

99. Golding JF, Markey HM, Stott JR. The effects of motion direction, body axis, and posture on motion sickness induced by low frequency linear oscillation. Aviat Space Environ Med (1995) 66(11):1046-51.

100. Schubert G. Über die physiologischen auswirkungen der corioliskräfte bei trudelbewegung des flugzeuges. vorläufige mitteilung. Acta Otolaryngol (1931) 16:39-47. doi:10.3109/00016483109122298

101. Gillingham KK. The spatial disorientation problem in the United States Air Force. J Vestib Res (1992) 2(4):297-306

102. Mateus J, Canizales J, Hearn AN, Young LR. Asymmetry in vestibular responses to cross-coupled stimulus. Exp Brain Res (2011) 209(4):561-9. doi:10.1007/s00221-011-2588-0

103. Guedry FE, Montague EK. Relationship between magnitudes of vestibular reactions and effective Coriolis couples in the semicircular canal system. Aerosp Med (1961) 32:487-500.

104. Jones GM. Origin significance and amelioration of coriolis illusions from the semicircular canals: a nonmathematical appraisal. Aerosp Med (1970) 41:483-90.

105. Lackner JR, Graybiel A. The effective intensity of Coriolis, cross-coupling stimulation is gravitoinertial force dependent: implications for space motion sickness. Aviat Space Environ Med (1986) 57(3):229-35.

106. Miller EF II, Graybiel A. Altered susceptibility to motion sickness as a function of subgravity level. Space Life Sci (1973) 4(2):295-306.

107. Miller EF II, Graybiel A. Experimental M-131 - human vestibular function. Aerosp Med (1973) 44(6):593-608.

108. Bockisch CJ, Straumann D, Haslwanter T. Eye movements during multi-axis whole-body rotations. J Neurophysiol (2003) 89(1):355-66. doi:10.1152/ jn.00058.2002

109. Grossman GE, Leigh RJ, Abel LA, Lanska DJ, Thurston SE. Frequency and velocity of rotational head perturbations during locomotion. Exp Brain Res (1988) 70(3):470-6. doi:10.1007/BF00247595

110. Cohen B, John P, Yakushin SB, Buettner-Ennever J, Raphan T. The nodulus and uvula: source of cerebellar control of spatial orientation of the angular vestibulo-ocular reflex. Ann N Y Acad Sci (2002) 978:28-45. doi:10.111 1/j.1749-6632.2002.tb07553.x

111. Wearne S, Raphan T, Cohen B. Control of spatial orientation of the angular vestibuloocular reflex by the nodulus and uvula. J Neurophysiol (1998) 79(5):2690-715.

112. Waespe W, Cohen B, Raphan T. Dynamic modification of the vestibuloocular reflex by the nodulus and uvula. Science (1985) 228(4696):199-202. doi:10.1126/science.3871968 
113. Clement G, Deguine O, Parant M, Costes-Salon MC, Vasseur-Clausen P, Pavy-LeTraon A. Effects of cosmonaut vestibular training on vestibular function prior to spaceflight. Eur J Appl Physiol (2001) 85(6):539-45. doi:10.1007/ s004210100494

114. Cheung CC, Hecht H, Jarchow T, Young LR. Threshold-based vestibular adaptation to cross-coupled canal stimulation. J Vestib Res (2007) 17(4): 171-81.

115. Clement G, Deguine O, Bourg M, Pavy-LeTraon A. Effects of vestibular training on motion sickness, nystagmus, and subjective vertical. J Vestib Res (2007) 17(5-6):227-37.

116. Cohen H, Cohen B, Raphan T, Waespe W. Habituation and adaptation of the vestibuloocular reflex: a model of differential control by the vestibulocerebellum. Exp Brain Res (1992) 90(3):526-38. doi:10.1007/BF00230935

117. Young LR, Hecht H, Lyne LE, Sienko KH, Cheung CC, Kavelaars J. Artificial gravity: head movements during short-radius centrifugation. Acta Astronaut (2001) 49(3-10):215-26. doi:10.1016/S0094-5765(01)00100-X

118. Bos JE, Bles W, de Graaf B. Eye movements to yaw, pitch, and roll about vertical and horizontal axes: adaptation and motion sickness. Aviat Space Environ Med (2002) 73(5):436-44.

119. Hoffer ME, Gottshall K, Kopke RD, Weisskopf P, Moore R, Allen KA, et al. Vestibular testing abnormalities in individuals with motion sickness. Otol Neurotol (2003) 24(4):633-6. doi:10.1097/00129492-200307000-00017

120. Dai M, Raphan T, Cohen B. Labyrinthine lesions and motion sickness susceptibility. Exp Brain Res (2007) 178(4):477-87. doi:10.1007/s00221-0060759-1

121. Dai M, Raphan T, Cohen B. Prolonged reduction of motion sickness sensitivity by visual-vestibular interaction. Exp Brain Res (2011) 210(3-4):503-13. doi:10.1007/s00221-011-2548-8

122. Cohen B, Dai M, Yakushin SB, Raphan T. Baclofen, motion sickness susceptibility and the neural basis for velocity storage. Prog Brain Res (2008) 171:543-53. doi:10.1016/S0079-6123(08)00677-8

123. Lackner JR, DiZio P. Human orientation and movement control in weightless and artificial gravity environments. Exp Brain Res (2000) 130(1):2-26. doi:10.1007/s002210050002

124. Tweed D, Fetter M, Sievering D, Misslisch H, Koenig E. Rotational kinematics of the human vestibuloocular reflex. II. Velocity steps. J Neurophysiol (1994) 72(5):2480-9.

125. Bertolini G, Bockisch CJ, Straumann D, Zee DS, Ramat S. Do humans show velocity-storage in the vertical rVOR? Prog Brain Res (2008) 171:207-10. doi:10.1016/S0079-6123(08)00628-6

126. Bertolini G, Ramat S. Velocity storage in the human vertical rotational vestibulo-ocular reflex. Exp Brain Res (2011) 209(1):51-63. doi:10.1007/ s00221-010-2518-6

127. Colagiorgio P, Bertolini G, Bockisch CJ, Straumann D, Ramat S. Multiple timescales in the adaptation of the rotational VOR. J Neurophysiol (2015) 113(9):3130-42. doi:10.1152/jn.00688.2014

128. Lawther A, Griffin MJ. The motion of a ship at sea and the consequent motion sickness amongst passengers. Ergonomics (1986) 29(4):535-52. doi:10.1080/00140138608968289

129. Lawther A, Griffin MJ. Prediction of the incidence of motion sickness from the magnitude, frequency, and duration of vertical oscillation. J Acoust Soc Am (1987) 82(3):957-66. doi:10.1121/1.395295

130. Alexander SJ, Cotzin M, Klee JB, Wendt GJ. Studies of motion sickness; the effects upon sickness rates of waves of various frequencies but identical acceleration. J Exp Psychol (1947) 37(5):440-8. doi:10.1037/h0063240

131. O'Hanlon JF, McCauley ME. Motion sickness incidence as a function of the frequency and acceleration of vertical sinusoidal motion. Aerosp Med (1974) 45(4):366-9.

132. McCauley ME, Royal JW, Wylie CD, O’Hanlon JF, Mackie RR. Motion Sickness Incidence: Exploratory Studies of Habituation, Pitch and Roll and the Refinement of a Mathematical Model. Goeleta, CA: Human Factor Research Inc. (1976). Contract No.: Technical Report 1733-2.

133. Griffin MJ. Handbook of Human Vibration. London: Academic Press (1990).

134. Joseph JA, Griffin MJ. Motion sickness: effect of changes in magnitude of combined lateral and roll oscillation. Aviat Space Environ Med (2008) 79(11):1019-27. doi:10.3357/ASEM.2196.2008

135. Beard GF, Griffin MJ. Discomfort caused by low-frequency lateral oscillation, roll oscillation and roll-compensated lateral oscillation. Ergonomics (2013) 56(1):103-14. doi:10.1080/00140139.2012.729613
136. Golding JF, Mueller AG, Gresty MA. A motion sickness maximum around the $0.2 \mathrm{~Hz}$ frequency range of horizontal translational oscillation. Aviat Space Environ Med (2001) 72(3):188-92.

137. Golding JF, Markey HM. Effect of frequency of horizontal linear oscillation on motion sickness and somatogravic illusion. Aviat Space Environ Med (1996) 67(2):121-6.

138. Wyllie IH, Griffin MJ. Discomfort from sinusoidal oscillation in the roll and lateral axes at frequencies between 0.2 and 1.6 Hz. J Acoust Soc Am (2007) 121(5 Pt1):2644-54. doi:10.1121/1.2715654

139. Donohew BE, Griffin MJ. Motion sickness with fully roll-compensated lateral oscillation: effect of oscillation frequency. Aviat Space Environ Med (2009) 80(2):94-101. doi:10.3357/ASEM.2345.2009

140. Donohew BE, Griffin MJ. Motion sickness: effect of the frequency of lateral oscillation. Aviat Space Environ Med (2004) 75(8):649-56.

141. Griffin MJ, Mills KL. Effect of frequency and direction of horizontal oscillation on motion sickness. Aviat Space Environ Med (2002) 73(6):537-43.

142. Cheung B, Nakashima A, RESEARCH D, TORONTO D. A Review on the Effects of Frequency of Oscillation on Motion Sickness. Toronto: Defense Technical Information Center (2006).

143. Bos JE. Less sickness with more motion and/or mental distraction. J Vestibul Res (2015) 25(1):23-33. doi:10.3233/Ves-150541

144. Khalid H, Turan O, Bos JE. Theory of a subjective vertical-horizontal conflict physiological motion sickness model for contemporary ships. JMar Sci Technol (2011) 16(2):214-25. doi:10.1007/S00773-010-0113-Y

145. Khalid H, Turan O, Bos JE, Incecik A. Application of the subjective vertical-horizontal-conflict physiological motion sickness model to the field trials of contemporary vessels. Ocean Eng (2011) 38(1):22-33. doi:10.1016/j. oceaneng.2010.09.008

146. Wiker SF, Kennedy RS, McCauley ME, Pepper RL. Susceptibility to seasickness: influence of hull design and steaming direction. Aviat Space Environ Med (1979) 50(10):1046-51.

147. Wertheim AH, Bos JE, Bles W. Contributions of roll and pitch to sea sickness. Brain Res Bull (1998) 47(5):517-24. doi:10.1016/S0361-9230(98)00098-7

148. Morton G, Cipriani A, Mc ED. Mechanism of motion sickness. Arch Neurol Psychiatry (1947) 57(1):58-70. doi:10.1001/archneurpsyc.1947. 02300240074003

149. Howarth HV, Griffin MJ. Effect of roll oscillation frequency on motion sickness. Aviat Space Environ Med (2003) 74(4):326-31.

150. Ueno M, Ogawa T, Nakagiri S, Arisawa T, Mino Y, Oyama K, et al. Studies on motion sickness caused by high curve speed railway vehicles. Evaluation of the swing and its effects on passengers and conductors. Sangyo Igaku (1986) 28(4):266-74. doi:10.1539/joh1959.28.266

151. Donohew BE, Griffin MJ. Motion sickness with combined lateral and roll oscillation: effect of percentage compensation. Aviat Space Environ Med (2010) 81(1):22-9. doi:10.3357/ASEM.2555.2010

152. Persson R. Motion Sickness in Tilting Trains. Description and Analysis of Present Knowledge. Linkoping: VTI (2008). Contract No.: VTI report 614A

153. Joseph JA, Griffin MJ. Motion sickness from combined lateral and roll oscillation: effect of varying phase relationships. Aviat Space Environ Med (2007) 78(10):944-50. doi:10.3357/ASEM.2043.2007

154. Reason J. An Investigation of Some Factors Contributing to Individual Variations in Motion Sickness Susceptibility. London: Ministry of Defence (Air) (1968). Contract No.: Flying Personnel Research Committee Report No. 1277.

155. Turner M, Griffin MJ. Motion sickness in public road transport: passenger behavior and susceptibility. Ergonomics (1999) 42(3):444-61. doi:10.1080/001401399185586

156. Griffin MJ, Newman MM. An experimental study of low-frequency motion in cars. J Automob Eng (2004) 218(11):1231-8. doi:10.1243/0954407042580093

157. Vogel H, Kohlhaas R, von Baumgarten RJ. Dependence of motion sickness in automobiles on the direction of linear acceleration. Eur J Appl Physiol Occup Physiol (1982) 48(3):399-405. doi:10.1007/BF00430230

158. Turner M, Griffin MJ. Motion sickness in public road transport: the effect of driver, route and vehicle. Ergonomics (1999) 42(12):1646-64. doi:10.1080/001401399184730

159. Golding JF, Bles W, Bos JE, Haynes T, Gresty MA. Motion sickness and tilts of the inertial force environment: active suspension systems vs. active passengers. Aviat Space Environ Med (2003) 74(3):220-7.

160. KoppaRJ,HayesGG.Driverinputsduringemergencyorextremevehiclemaneuvers. Hum Factors (1976) 18(4):361-9. doi:10.1177/001872087601800405 
161. Rolnick A, Lubow RE. Why is the driver rarely motion sick - the role of controllability in motion sickness. Ergonomics (1991) 34(7):867-79. doi:10.1080/00140139108964831

162. Perrin P, Lion A, Bosser G, Gauchard G, Meistelman C. Motion sickness in rally car co-drivers. Aviat Space Environ Med (2013) 84(5):473-7. doi:10.3357/ASEM.3523.2013

163. Wada T, Konno H, Fujisawa S, Doi S. Can passengers' active head tilt decrease the severity of carsickness? Effect of head tilt on severity of motion sickness in a lateral acceleration environment. Hum Factors (2012) 54(2):226-34. doi:10.1177/0018720812436584

164. Wada T, Yoshida K. Effect of passengers' active head tilt and opening/closure of eyes on motion sickness in lateral acceleration environment of cars. Ergonomics (2015):1-26. doi:10.1080/00140139.2015.1109713

165. Schoettle B, Sivak M. Public Opinion about Self-Driving Vehicles in China, India, Japan, the U.S., the U.K., and Australia. Ann Arbor: The University of Michigan Transportation Research Institute: Transportation Research Institute (2014). (Report No. UMTRI-2014-30)

166. Diels C, Bos JE. Self-driving carsickness. Appl Ergon (2015) 53(Pt B):374-82. doi:10.1016/j.apergo.2015.09.009

Conflict of Interest Statement: The authors declare that the research was conducted in the absence of any commercial or financial relationships that could be construed as a potential conflict of interest.

Copyright (C) 2016 Bertolini and Straumann. This is an open-access article distributed under the terms of the Creative Commons Attribution License (CC BY). The use, distribution or reproduction in other forums is permitted, provided the original author(s) or licensor are credited and that the original publication in this journal is cited, in accordance with accepted academic practice. No use, distribution or reproduction is permitted which does not comply with these terms. 